KYUSHU-HET 61

STUPP-02-170

\title{
Yet another correlation in the analysis of CP violation using a neutrino oscillation experiment
}

\author{
Toshihiko Ota ${ }^{1}$ and Joe Sato ${ }^{2}$, \\ ${ }^{1}$ Department of Physics, Kyushu University, \\ Hakozaki, Higashi-ku, Fukuoka 812-8581, Japan \\ ${ }^{2}$ Department of Physics, Faculty of Science, \\ Saitama University, Saitama, 338-85\%0, Japan
}

\begin{abstract}
We investigate the effect induced by variations in the density profile of the Earth's interior using a long-baseline neutrino oscillation experiment.

At first, we point out two facts. (i) The most essential part of the matter profile is the first Fourier mode of the matter profile function; and (ii) The Earth models based on the study of seismology include a large uncertainty for the first Fourier mode. Next, we show that there is a strong correlation between the average density value and the first Fourier coefficient in the analysis of oscillation probability. This means that the matter profile effect induces added uncertainty for the average matter parameter.

Taking into account this extra uncertainty, we make numerical calculations for the sensitivity to $\mathrm{CP}$ violation search and show that $\mathrm{CP}$ sensitivity is impaired by this added uncertainty within a large $U_{e 3}$ region.

PACS numbers: 13.15.+g, 14.60.Lm, 14.60.Pq, 91.35.-x

Keywords: neutrino oscillation, neutrino factory, matter effect, Earth matter density profile, degeneracy, correlation
\end{abstract}

\section{INTRODUCTION}

The effects of the Earth's matter [1] play a very important role in long-baseline and high-energy oscillation experiments, such as a neutrino factory [2]. Furthermore, the effect of variations in matter density and chemical composition along the baseline on the oscillation probability, which we call the matter profile effect, is also a controversial issue and has been debated in various contexts $33,4,5,6,6,8,9,10,11,12,13,14,15$.

In the search for $\mathrm{CP}$-violating effects, it is important to understand the structure of the Earth so as to estimate precisely the fake signal induced by the Earth's matter. Some research 13 has concluded that it is hard to obtain information about the interior of the Earth through neutrino experiments using the currently assumed size of statistics and realistic detector configuration. Therefore, the science of geophysics has been applied to support the analysis. Much analysis has used the Preliminary Reference Earth Model (PREM) [16], an Earth model which is based on seismology. When we deal with such a model, we need to be conscious of the fact that the model includes uncertainty. In this paper, we discuss the fact that this uncertainty reduces the sensitivity of the $\mathrm{CP}$ violation and we show that it is not a small effect.

The procedure used to demonstrate our argument is as follows: Firstly, we recapitulate the method of the Fourier series expansion of the matter profile function [14, 15] in Sec.11. Using this method, we show that only the first few modes are important in a high-energy experiment. Furthermore, we mention the uncertainty included in the seismological Earth models. We point out that a few percent error for the profile function can actually be interpreted as huge uncertainty for the Fourier coefficients, which can affect the CP sensitivity. Next, in Sec.III, we show that there is a strong correlation between the constant matter parameter and the first Fourier coefficient of the matter profile function within a wide energy and baseline region. This fact means that the uncertainty of the matter profile effect brings added uncertainty to the average matter density. Then, we present numerical calculations to show quantitatively a loss of sensitivity induced by the uncertainty of the matter profile effect in Sec. [V]. Finally, a summary is given in Sec. $\mathrm{V}$.

\section{METHOD OF THE FOURIER SERIES}

We would like to point out two facts in this section. (1) The most essential part of the matter profile effect is the first Fourier mode of the matter profile function. (2) The seismological Earth models include great uncertainty for the first Fourier mode. These facts force us to reconsider analyses in the baseline region which have so far assumed that the matter profile effect is not significant. In the case where the baseline length is 3,000 km, PREM tells us that the

*Electronic address: toshi@higgs.phys.kvushu-u.ac.jp

${ }^{\dagger}$ Electronic address: oe@phy.saitama-u.ac.jp 
matter profile function is almost flat and hence it can be expected that the matter profile effect is small. However, if we consider the uncertainty of PREM, how will the analysis change?

\section{A. Introduction of the method}

To see the effects induced by the matter profile, we derive the analytic expression using the method of the Fourier series 14, 15. Expanding the matter profile function into the Fourier modes, we obtain an extremely clear viewpoint for the resonance conditions between the oscillation lengths of the neutrino and the matter profile undulation. By this expansion we can understand which modes, and what structures, are effective*.

Now, we introduce our calculation method. We assume three generations and parameterize the mixing matrix of the lepton sector, Maki-Nakagawa-Sakata-Pontecorvo matrix (MNSP matrix), as follows;

$$
U_{\alpha i}=\left(\begin{array}{ccc}
1 & & \\
& c_{\psi} & s_{\psi} \\
& -s_{\psi} & c_{\psi}
\end{array}\right)\left(\begin{array}{ccc}
1 & & \\
& 1 & \\
& & e^{i \delta}
\end{array}\right)\left(\begin{array}{ccc}
c_{\phi} & & s_{\phi} \\
& 1 & \\
-s_{\phi} & & c_{\phi}
\end{array}\right)\left(\begin{array}{ccc}
c_{\omega} & s_{\omega} & \\
-s_{\omega} & c_{\omega} & \\
& & 1
\end{array}\right) U_{\text {Majorana }}, \quad(\alpha=e, \mu, \tau, \quad i=1,2,3)
$$

Here, $s$ and $c$ denote sin and cos, and $U_{\text {Majorana }}$ is the so-called Majorana phase matrix, which does not contribute to the oscillation phenomena. The effective Hamiltonian for neutrino propagation is

$$
H(x)_{\beta \alpha}=\frac{1}{2 E}\left\{U_{\beta i}\left(\begin{array}{lll}
0 & & \\
& \Delta m_{21}^{2} & \\
& & \Delta m_{31}^{2}
\end{array}\right) U_{i \alpha}^{\dagger}+\left(\begin{array}{ccc}
a_{0}+\delta a(x) & \\
& 0 & \\
& & 0
\end{array}\right)_{\beta \alpha}\right\}
$$

where $\Delta m_{i j}^{2}$ is the squared-mass difference between the $i$ th and $j$ th generations in a vacuum. We separate the matter effect into two parts, $a_{0}$ and $\delta a(x)$. The effect of the average matter is denoted by $a_{0}$, and $\delta a(x)$ is the matter profile part, that is, the deviation part from the average density which depends on the position, $x$. After the separation, we expand the matter profile part into the Fourier series,

$$
\delta a(x)=\sum_{\substack{n=-\infty \\ n \neq 0}}^{\infty} a_{n} e^{-i p_{n} x}, \quad p_{n} \equiv \frac{2 \pi}{L} n
$$

Note that the relation, $a_{n}=a_{-n}^{*}$ is always satisfied because of the condition that $\delta a(x)$ is real. For anti-neutrino, $a_{0}$, $a_{n}$ and $\delta$ should be replaced by $-a_{0},-a_{n}$ and $-\delta$, respectively.

We treat $\Delta m_{21}^{2}$ and $\delta a(x)$ as perturbations and obtain the oscillation probabilities up to the first order of them, for example [15],

$$
\begin{aligned}
& P_{\nu_{e} \rightarrow \nu_{\mu}}=s_{\psi}^{2} s_{2 \tilde{\phi}}^{2} \sin ^{2} \frac{\lambda_{+}-\lambda_{-}}{4 E} L \quad \quad \equiv P^{\text {main }} \\
& +\frac{1}{2} c_{\delta} s_{2 \psi} s_{2 \omega} s_{2 \tilde{\phi}} \\
& \times\left[\left(c_{\tilde{\phi}} c_{\phi-\tilde{\phi}} \frac{\Delta m_{21}^{2}}{\Delta m_{21}^{2} c_{\omega}^{2}-\lambda_{-}}+s_{\tilde{\phi}} s_{\phi-\tilde{\phi}} \frac{\Delta m_{21}^{2}}{\lambda_{+}-\Delta m_{21}^{2} c_{\omega}^{2}}\right) \sin ^{2} \frac{\Delta m_{21}^{2} c_{\omega}^{2}-\lambda_{-}}{4 E} L\right. \\
& -\left(c_{\tilde{\phi}} c_{\phi-\tilde{\phi}} \frac{\Delta m_{21}^{2}}{\Delta m_{21}^{2} c_{\omega}^{2}-\lambda_{-}}+s_{\tilde{\phi}} s_{\phi-\tilde{\phi}} \frac{\Delta m_{21}^{2}}{\lambda_{+}-\Delta m_{21}^{2} c_{\omega}^{2}}\right) \sin ^{2} \frac{\lambda_{+}-\Delta m_{21}^{2} c_{\omega}^{2}}{4 E} L \\
& \left.+\left(c_{\tilde{\phi}} c_{\phi-\tilde{\phi}} \frac{\Delta m_{21}^{2}}{\Delta m_{21}^{2} c_{\omega}^{2}-\lambda_{-}}-s_{\tilde{\phi}} s_{\phi-\tilde{\phi}} \frac{\Delta m_{21}^{2}}{\lambda_{+}-\Delta m_{21}^{2} c_{\omega}^{2}}\right) \sin ^{2} \frac{\lambda_{+}-\lambda_{-}}{4 E} L\right] \\
& +\frac{1}{4} s_{\delta} s_{2 \psi} s_{2 \omega} s_{2 \tilde{\phi}}\left(c_{\tilde{\phi}} c_{\phi-\tilde{\phi}} \frac{\Delta m_{21}^{2}}{\Delta m_{21}^{2} c_{\omega}^{2}-\lambda_{-}}+s_{\tilde{\phi}} s_{\phi-\tilde{\phi}} \frac{\Delta m_{21}^{2}}{\lambda_{+}-\Delta m_{21}^{2} c_{\omega}^{2}}\right) \\
& \times\left(\sin \frac{\lambda_{+}-\Delta m_{21}^{2} c_{\omega}^{2}}{2 E} L+\sin \frac{\Delta m_{21}^{2} c_{\omega}^{2}-\lambda_{-}}{2 E} L-\sin \frac{\lambda_{+}-\lambda_{-}}{2 E} L\right) \\
& +4 s_{\psi}^{2} s_{2 \tilde{\phi}}^{2} c_{2 \tilde{\phi}} \sum_{n=1}^{\infty} \operatorname{Re}\left[a_{n}\right] \frac{\lambda_{+}-\lambda_{-}}{\left(\lambda_{+}-\lambda_{-}\right)^{2}-\left(2 E p_{n}\right)^{2}} \sin ^{2} \frac{\lambda_{+}-\lambda_{-}}{4 E} L \\
& P_{\nu_{e} \rightarrow \nu_{\tau}}=c_{\psi}^{2} s_{2 \tilde{\phi}}^{2} \sin ^{2} \frac{\lambda_{+}-\lambda_{-}}{4 E} L \\
& \equiv \sum_{n=1}^{\infty} P_{n}^{\text {profile }}
\end{aligned}
$$

\footnotetext{
* The Fourier expanded-matter profile can not reproduce the boundary between two layers precisely. However, it will be shown below that the higher modes which construct the edge are not effective in high-energy experiments.
} 


$$
\begin{aligned}
& -\frac{1}{2} c_{\delta} s_{2 \psi} s_{2 \omega} s_{2 \tilde{\phi}} \\
& \times\left[\left(c_{\tilde{\phi}} c_{\phi-\tilde{\phi}} \frac{\Delta m_{21}^{2}}{\Delta m_{21}^{2} c_{\omega}^{2}-\lambda_{-}}+s_{\tilde{\phi}} s_{\phi-\tilde{\phi}} \frac{\Delta m_{21}^{2}}{\lambda_{+}-\Delta m_{21}^{2} c_{\omega}^{2}}\right) \sin ^{2} \frac{\Delta m_{21}^{2} c_{\omega}^{2}-\lambda_{-}}{4 E} L\right. \\
& -\left(c_{\tilde{\phi}} c_{\phi-\tilde{\phi}} \frac{\Delta m_{21}^{2}}{\Delta m_{21}^{2} c_{\omega}^{2}-\lambda_{-}}+s_{\tilde{\phi}} s_{\phi-\tilde{\phi}} \frac{\Delta m_{21}^{2}}{\lambda_{+}-\Delta m_{21}^{2} c_{\omega}^{2}}\right) \sin ^{2} \frac{\lambda_{+}-\Delta m_{21}^{2} c_{\omega}^{2}}{4 E} L \\
& \left.+\left(c_{\tilde{\phi}} c_{\phi-\tilde{\phi}} \frac{\Delta m_{21}^{2}}{\Delta m_{21}^{2} c_{\omega}^{2}-\lambda_{-}}-s_{\tilde{\phi}} s_{\phi-\tilde{\phi}} \frac{\Delta m_{21}^{2}}{\lambda_{+}-\Delta m_{21}^{2} c_{\omega}^{2}}\right) \sin ^{2} \frac{\lambda_{+}-\lambda_{-}}{4 E} L\right] \\
& -\frac{1}{4} s_{\delta} s_{2 \psi} s_{2 \omega} s_{2 \tilde{\phi}}\left(c_{\tilde{\phi}} c_{\phi-\tilde{\phi}} \frac{\Delta m_{21}^{2}}{\Delta m_{21}^{2} c_{\omega}^{2}-\lambda_{-}}+s_{\tilde{\phi}} s_{\phi-\tilde{\phi}} \frac{\Delta m_{21}^{2}}{\lambda_{+}-\Delta m_{21}^{2} c_{\omega}^{2}}\right) \\
& \times\left(\sin \frac{\lambda_{+}-\Delta m_{21}^{2} c_{\omega}^{2}}{2 E} L+\sin \frac{\Delta m_{21}^{2} c_{\omega}^{2}-\lambda_{-}}{2 E} L-\sin \frac{\lambda_{+}-\lambda_{-}}{2 E} L\right) \\
& +4 c_{\psi}^{2} s_{2 \tilde{\phi}}^{2} c_{2 \tilde{\phi}} \sum_{n=1}^{\infty} \operatorname{Re}\left[a_{n}\right] \frac{\lambda_{+}-\lambda_{-}}{\left(\lambda_{+}-\lambda_{-}\right)^{2}-\left(2 E p_{n}\right)^{2}} \sin ^{2} \frac{\lambda_{+}-\lambda_{-}}{4 E} L, \\
& P_{\nu_{e} \rightarrow \nu_{e}}=1-s_{2 \tilde{\phi}}^{2} \sin ^{2} \frac{\lambda_{+}-\lambda_{-}}{4 E} L \\
& -4 s_{2 \tilde{\phi}}^{2} c_{2 \tilde{\phi}} \sum_{n=1}^{\infty} \operatorname{Re}\left[a_{n}\right] \frac{\lambda_{+}-\lambda_{-}}{\left(\lambda_{+}-\lambda_{-}\right)^{2}-\left(2 E p_{n}\right)^{2}} \sin ^{2} \frac{\lambda_{+}-\lambda_{-}}{4 E} L .
\end{aligned}
$$

Here the effective mixing angle in the average density of the matter, $\tilde{\phi}$, and the squared-mass eigenvalue, $\lambda_{ \pm}$, in the average density are given by

$$
\begin{gathered}
\tan 2 \tilde{\phi}=\frac{s_{2 \phi}\left(\Delta m_{31}^{2}-\Delta m_{21}^{2} s_{\omega}^{2}\right)}{c_{2 \phi}\left(\Delta m_{31}^{2}-\Delta m_{21}^{2} s_{\omega}^{2}\right)-a_{0}}, \\
\lambda_{ \pm}=\frac{1}{2}\left[\Delta m_{31}^{2}+\Delta m_{21}^{2} s_{\omega}^{2}+a_{0} \pm \sqrt{\left\{\left(\Delta m_{31}^{2}-\Delta m_{21}^{2} s_{\omega}^{2}\right) c_{2 \phi}-a_{0}\right\}^{2}+\left(\Delta m_{31}^{2}-\Delta m_{21}^{2} s_{\omega}^{2}\right)^{2} s_{2 \phi}^{2}}\right] .
\end{gathered}
$$

Note that the effects of the asymmetric profile do not appear in the first order of the perturbations.

The resonance conditions, $\lambda_{+}-\lambda_{-}=2 E p_{n}$, show the energy range and width of the resonance induced by each Fourier mode. We understand that the higher Fourier mode can resonate only with the lower energy neutrinos whose oscillation lengths are shorter [3]. Furthermore, the half-width of the amplitude becomes narrow as the mode becomes higher. This means that if the lower energy neutrino were to be observed precisely, the fine structure of the Earth could be known, although it is actually extremely difficult to achieve such observation. Therefore, we conclude that only the first few Fourier modes, which are determined by the large structure of the matter profile, are relevant in the currently assumed experimental set-ups. This is consistent with the results obtained using different methods [11, 12, 13].

\section{B. Uncertainty of the Earth model}

Knowledge of geophysics is essential since it is very difficult to ascertain the profile of the Earth from a neutrino experiment. So far, PREM has been regarded as the absolute model. We have tended to expect that the error and the effect induced by the error are so small that they can be neglected without a careful consideration, although we have to estimate how much error the model includes in order to use the seismological Earth model. In order to discuss this error, we introduce another Earth model, ak135-f [17].

Figure 1 represents the matter profile function calculated using PREM (solid line) and ak135-f (dotted line) in the case where the baseline length is $3,000 \mathrm{~km}$ and $7,332 \mathrm{~km}$, respectively. The profile function based on PREM is almost flat at $L=3,000 \mathrm{~km}$, and this fact guarantees that the matter profile effect is small. According to ak135-f, however, it is not so flat. If we follow this model, we may be unable to ignore the matter profile effect, even at this baseline length. Furthermore, the authors of Ref.[17] note that "the upper mantle density model should be treated with caution and may well change with further work." The upper mantle and transition area (up to $670 \mathrm{~km}$ in depth) occupy a large part of the path of the neutrino beam in the case of $L=3,000 \mathrm{~km}$.

The baseline dependence of the average density and the first Fourier coefficient are compared in Fig.2. These two models are different in terms of the Fourier coefficient, although they are similar with regard to the average matter density. In particular, around $L=3,000 \mathrm{~km}$, the difference of the first Fourier coefficient is quite large. We need to recognize that seismological models include uncertainty, the size of which is at least equal to the difference in these 

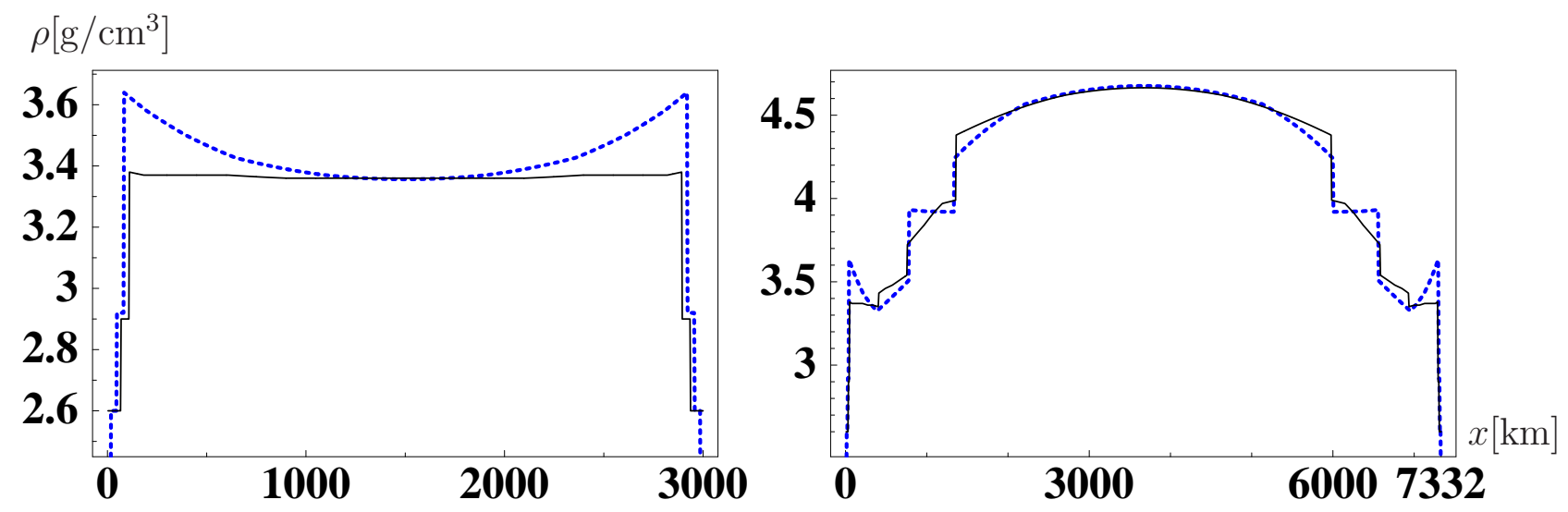

FIG. 1: The matter profile functions in the case of 3,000 km and 7,332 km which are calculated using PREM (solid line) and ak135-f (dotted line).
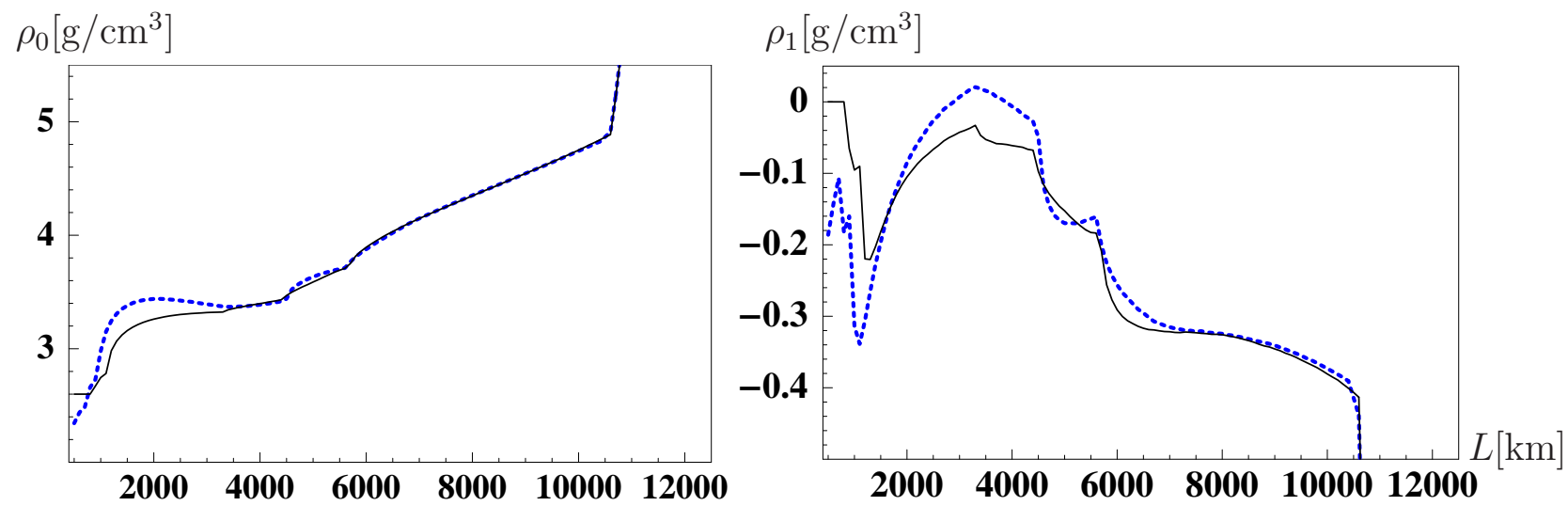

FIG. 2: The baseline dependence of the average matter density and the first Fourier coefficient which are calculated using PREM (solid line) and ak135-f (dotted line). Around $L=3,000 \mathrm{~km}$, the average densities of these two models are almost the same within a few percent, but the first Fourier coefficients differ by more than $100 \%$.

two models ${ }^{\dagger}$. The authors of Ref.[18] state that $\pm 2 \sim 3 \%$ uncertainty in the upper mantle is a reasonable estimation, which produces a large, as much as $100 \%$, uncertainty for the first Fourier coefficient. If $2.5 \%$ error for PREM is allowed, we can assume the matter profile depicted Fig.3 instead of PREM itself. This profile realizes $100 \%$ shift of the first Fourier coefficient without adjusting the average matter density. We would like to stress again that the uncertainty of the average density may indeed be small, but the uncertainty of the profile is not so small that it can be neglected, especially in $L \simeq 3,000 \mathrm{~km}$.

\section{CORRELATION BETWEEN AVERAGE MATTER PARAMETER AND FIRST FOURIER COEFFICIENT}

Here, we will establish the existence of the correlation between the parameter for average matter density and the first Fourier coefficient of the matter profile function. The effect induced by the matter profile and its uncertainty can be understood by the concept of this correlation. Although the average density can be determined at a few percent accuracy through studies of the seismic wave and gravitational effect etc., the matter profile effect adds extra uncertainty to the average matter density. As we pointed out in the previous section, the uncertainty of the first Fourier mode is not small, especially in the case where the neutrino beam passes mainly through the upper mantle and the transition zone, namely where the baseline length is around 3,000 km.

\section{A. One example: $L=7,332 \mathrm{~km}$}

To corroborate that the correlation exists, we first present an example. In Fig. 目, the oscillation probability for $\nu_{e} \rightarrow \nu_{\mu}$ is shown where the baseline length is $7,332 \mathrm{~km}$. The solid line is calculated using the full-PREM profile. As

\footnotetext{
$\dagger$ Assumption for the profile of the underground chemical component is made when the density profile is determined in geophysics. This assumption affects the density of the electron number that we really want to ascertain.
} 


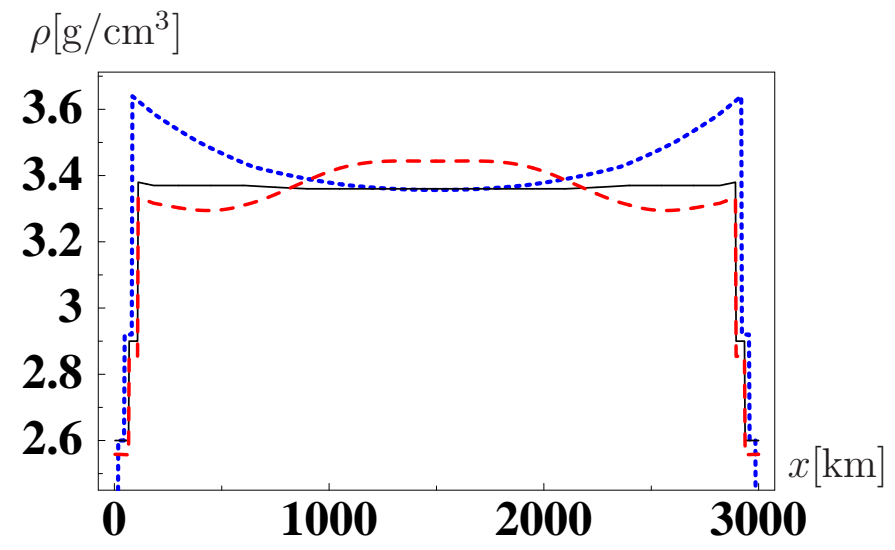

FIG. 3: An example where the deviation of the first Fourier coefficient from PREM is more than $100 \%$ in $L=3,000 \mathrm{~km}($ dashed line). This is drawn by modifying PREM within $2.5 \%$. The first Fourier coefficient for this profile is $-0.084 \mathrm{~g} / \mathrm{cm}^{3}, \mathrm{which}$ is almost twice as large as that of PREM, $-0.043 \mathrm{~g} / \mathrm{cm}^{3}$. Solid and dotted lines are the same as those in Fig.1.

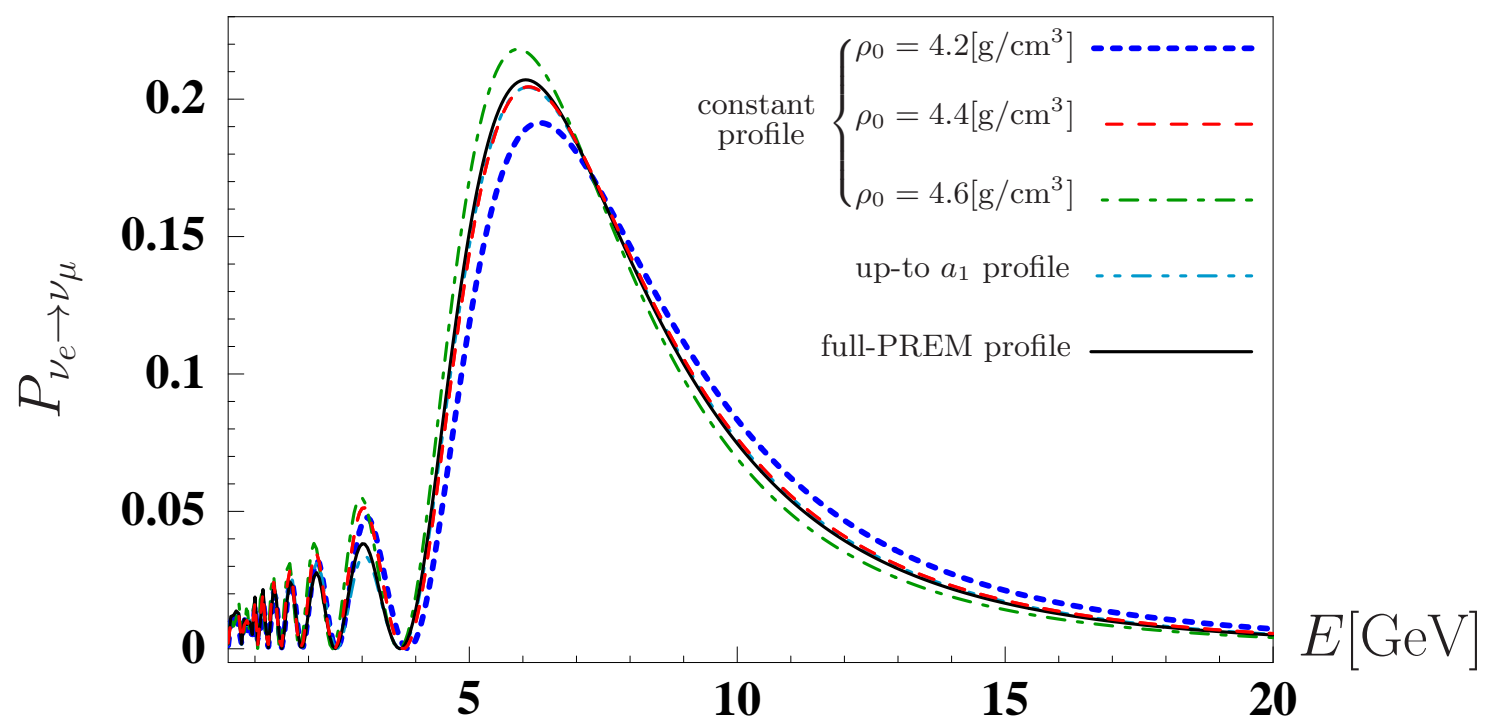

FIG. 4: Oscillation probability for $\nu_{e} \rightarrow \nu_{\mu}$ in $L=7,332 \mathrm{~km}$. The dotted, dashed, and dash-dot lines are calculated using the constant matter profile where the values of the matter parameter are $4.2,4.4$, and $4.6 \mathrm{~g} / \mathrm{cm}^{3}$, respectively. The dash-dot-dot line is calculated by the up-to-first-mode profile where the values are $\rho_{0}=4.2 \mathrm{~g} / \mathrm{cm}^{3}$ and $\rho_{1}=-0.32 \mathrm{~g} / \mathrm{cm}^{3}$. The solid line uses the full-PREM matter profile, and this line is quite similar to the dash-dot-dot and dashed lines. We set the oscillation parameters as $\sin \omega=0.5, \sin \psi=1 / \sqrt{2}, \sin \phi=0.1, \Delta m_{31}^{2}=2.5 \times 10^{-3} \mathrm{eV}^{2}, \Delta m_{21}^{2}=5.0 \times 10^{-5} \mathrm{eV}^{2}$, and $\delta=\pi / 2$.

we showed in Ref.[15], this line is almost the same as the dash-dot-dot line which is calculated by the up-to-first-mode profile with $\rho_{0}=4.2 \mathrm{~g} / \mathrm{cm}^{3}$ and $\rho_{1}=-0.32 \mathrm{~g} / \mathrm{cm}^{3}$, whose values are based on PREM.

The dotted, dashed, and dash-dot lines are those which are calculated using the constant-matter profile where the average density is $4.2,4.4$, and $4.6 \mathrm{~g} / \mathrm{cm}^{3}$, respectively. The PREM tells us that the average density in this baseline length is $4.2 \mathrm{~g} / \mathrm{cm}^{3}$. This figure shows that the constant profile with the average density following PREM (dotted line) cannot reproduce the behavior of the oscillation probability with the full-PREM profile (solid line). However, Fig. 1 also shows that if a shift in the constant value is allowed, then a good fit with the full-PREM calculation can be realized at $\rho_{0}=4.4 \mathrm{~g} / \mathrm{cm}^{3}$. This fact means that the shift of the constant matter parameter can copy the matter profile effect, which is almost equal to the effect induced by the first Fourier modes with this baseline length. We can expect that there is a strong correlation between the parameter for the average matter density and the first Fourier coefficient of the matter profile function. Indeed, this correlation is not an accidental phenomenon in this example.

We now consider the mechanism on which this correlation is based. The existence of the correlation suggests that the common shift in the average matter parameter over a wide energy region can imitate the effect of the first Fourier mode. This statement means that the relation,

$$
\frac{\partial P\left(a_{n}=0\right)}{\partial a_{0}} \Delta a_{0}=\sum_{n=1}^{\infty} P_{n}^{\text {profile }},
$$

is satisfied by the constant $\Delta a_{0}$ over a wide energy region. In the above example, the constant shift $\Delta \rho_{0}=0.2 \mathrm{~g} / \mathrm{cm}^{3}$ works well within the energy region above $4 \mathrm{GeV}$. Since we can assume that the unperturbed term of the oscillation probability is dominant over the other perturbative terms and that the matter profile effect can be represented by the 
first mode, the condition in eq.(9) reduces to

$$
\begin{gathered}
\frac{\partial P^{\text {main }}}{\partial a_{0}} \Delta a_{0}=P_{1}^{\text {profile }} \\
\Longleftrightarrow \frac{\Delta a_{0}}{\lambda_{+}-\lambda_{-}} \sin \frac{\lambda_{+}-\lambda_{-}}{4 E} L-\left(\frac{\Delta a_{0}}{4 E} L\right) \cos \frac{\lambda_{+}-\lambda_{-}}{4 E} L=2\left(\frac{\operatorname{Re}\left[a_{1}\right]}{4 E} L\right) \frac{\frac{\lambda_{+}-\lambda_{-}}{4 E} L}{\left(\frac{\lambda_{+}-\lambda_{-}}{4 E} L\right)^{2}-\pi^{2}} \sin \frac{\lambda_{+}-\lambda_{-}}{4 E} L .
\end{gathered}
$$

It is not inconsequential whether or not the constant shift $\Delta a_{0}$ can maintain the relation. To clarify this issue, we divide the energy region into two regions where $\frac{\lambda_{+}-\lambda_{-}}{4 E} L \sim 0$ and $\frac{\lambda_{+}-\lambda_{-}}{4 E} L \sim \frac{\pi}{2}$ are satisfied, and we investigate each region.

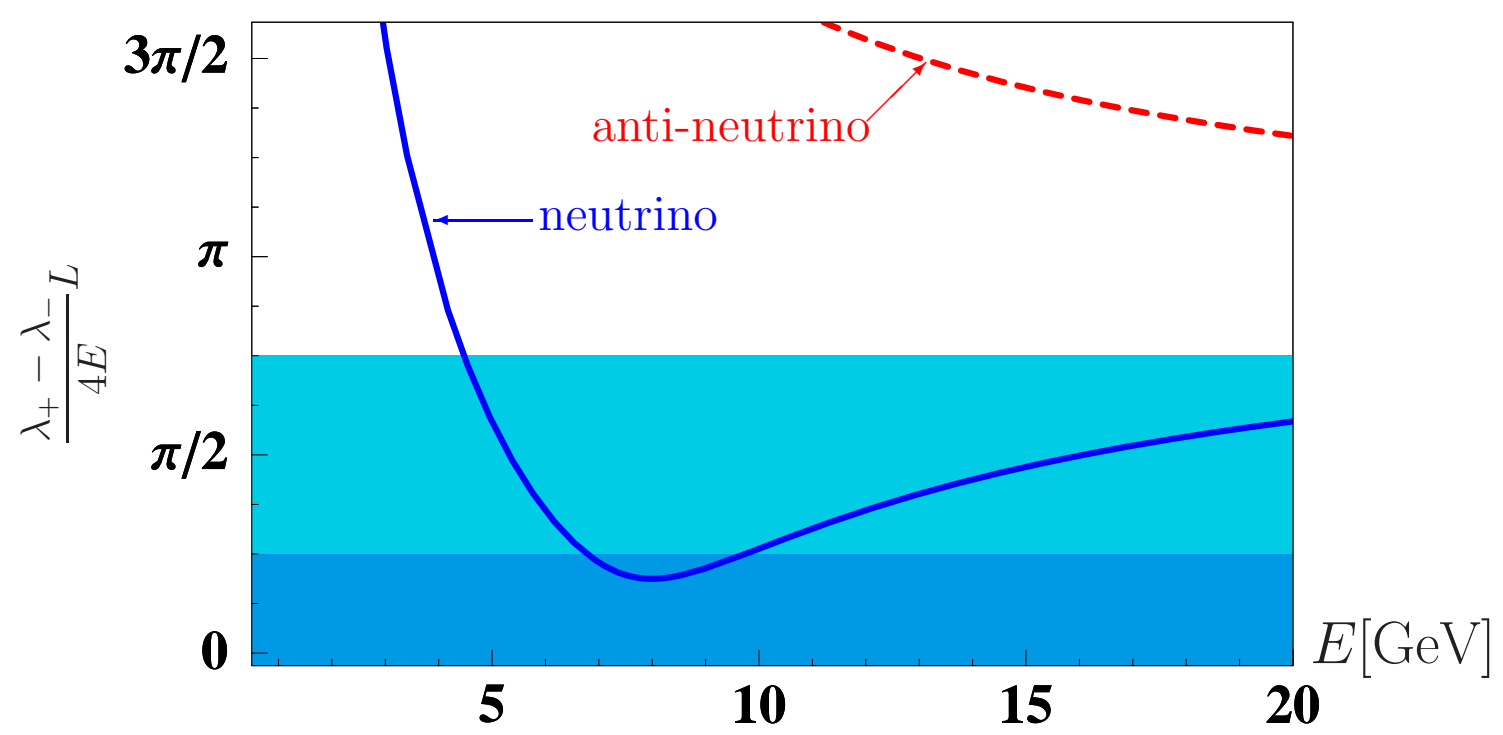

FIG. 5: Energy dependence of $\frac{\lambda_{+}-\lambda_{-}}{4 E} L$ for neutrino (solid line) and anti-neutrino (dashed line) in $L=7,332 \mathrm{~km}$. The oscillation parameters used in this figure are the same as those used in Fig. 4 .

Figure 0 shows the energy dependence of $\frac{\lambda_{+}-\lambda_{-}}{4 E} L$ corresponding to the case of Fig. In the region of $\frac{\lambda_{+}-\lambda_{-}}{4 E} L \sim 0$, the condition eq.(10) is approximated to

$$
\Delta a_{0}=-\frac{6}{\pi^{2}} \operatorname{Re}\left[a_{1}\right]+\mathcal{O}\left\{\left(\frac{\lambda_{+}-\lambda_{-}}{4 E} L\right)^{2}\right\} .
$$

Using the value $\rho_{1}=-0.32 \mathrm{~g} / \mathrm{cm}^{3}$, we understand from eq.(11) that shifting $\rho_{0}$ by $0.2 \mathrm{~g} / \mathrm{cm}^{3}$ makes the oscillation probability calculated with the constant profile clearly mimic the real oscillation probability, including the matter profile. Within the $\frac{\lambda_{+}-\lambda_{-}}{4 E} L \sim \frac{\pi}{2}$ region, the condition eq.(10) is approximated by

$$
\Delta a_{0}=-\frac{2}{3} \operatorname{Re}\left[a_{1}\right]+\mathcal{O}\left(\frac{\lambda_{+}-\lambda_{-}}{4 E} L-\frac{\pi}{2}\right) .
$$

This means that the shift of $a_{0}$ so as to mimic the matter profile effect is also about $0.2 \mathrm{~g} / \mathrm{cm}^{3}$, which is the same as the shift in the $\frac{\lambda_{+}-\lambda_{-}}{4 E} L \sim 0$ region. Therefore, in a wide energy region, such as both the $\frac{\lambda_{+}-\lambda_{-}}{4 E} L \sim 0$ and $\frac{\lambda_{+}-\lambda_{-}}{4 E} L \sim \frac{\pi}{2}$ regions, the common shift of $a_{0}$ can indeed copy the effect of the first Fourier mode ${ }^{\ddagger}$.

It is an essential point for the existence of the correlation that an experiment is only sensitive to these two energy ranges $^{\S}$. We note that the condition for anti-neutrino is the same as that for neutrino. Therefore, even if the analysis is made using both neutrino and anti-neutrino, the correlation still exists if the observed energy region for both neutrino and anti-neutrino satisfies either $\frac{\lambda_{+}-\lambda_{-}}{4 E} L \sim 0$ or $\frac{\lambda_{+}-\lambda_{-}}{4 E} L \sim \frac{\pi}{2}$. In the case of $L=7,332 \mathrm{~km}$, the energy range for anti-neutrino is not around either $\frac{\lambda_{+}-\lambda_{-}}{4 E} L \sim 0$ or $\frac{\lambda_{+}-\lambda_{-}}{4 E} L \sim \frac{\pi}{2}$, so if we could observe an adequate number of anti-neutrino events, the correlation would cease to hold, although the anti-neutrino event can be expected to be too short to be significant in a statistical sense. Therefore, we can conclude that the correlation still exists for this baseline length.

\footnotetext{
$¥$ Moreover, we can see that this common shift works well in the intermediate region by expanding the oscillation probability around $\frac{\lambda_{+}-\lambda_{-}}{4 E} L \sim \frac{\pi}{4}$.

$\S$ In the region where $\frac{\lambda_{+}-\lambda_{-}}{4 E} L \sim \pi$ holds, the relation becomes $\Delta a_{0}=-\frac{\pi}{\pi+1} \operatorname{Re}\left[a_{1}\right]+\mathcal{O}\left(\frac{\lambda_{+}-\lambda_{-}}{4 E} L-\pi\right)$, and hence the required shift to mimic the matter profile effect is significantly different.
} 


\section{B. Baseline region where the correlation exists}

There is a strong correlation between $a_{0}$ and $a_{1}$ within the energy and baseline region where $\frac{\lambda_{+}-\lambda_{-}}{4 E} L=0, \frac{\pi}{2}$ are satisfied, as we have established in the previous sub-section. The energy and baseline dependence of $\frac{\lambda_{+}-\lambda_{-}}{4 E} L$ for
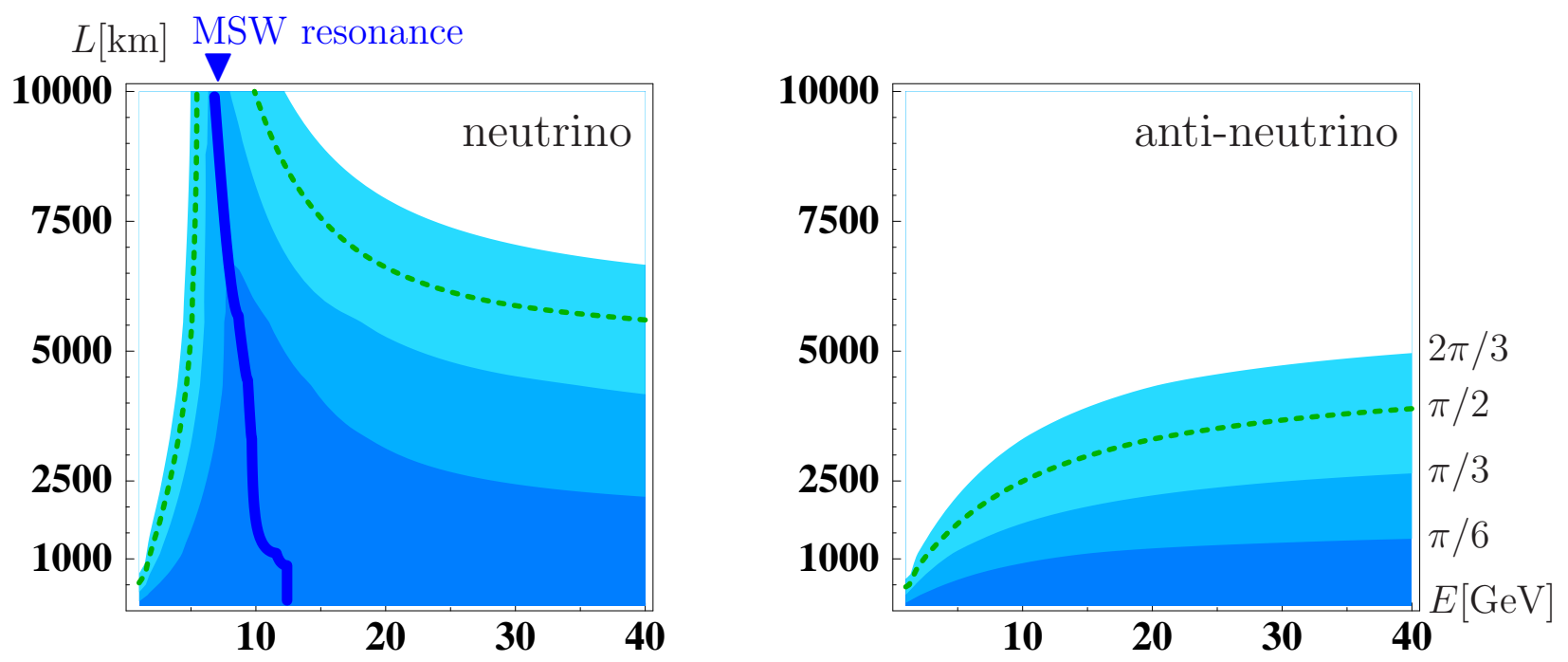

FIG. 6: Energy and baseline dependence of $\frac{\lambda_{+}-\lambda_{-}}{4 E} L$ for neutrino (left plot) and anti-neutrino (right plot). The reference values of the parameters are the same as in Fig.4.

neutrino and anti-neutrino is shown in Fig.6. This figure tells us the region where the correlation exists. In $L \lesssim 5,000$ $\mathrm{km}, \frac{\lambda_{+}-\lambda_{-}}{4 E} L$ is near 0 or $\frac{\pi}{2}$ for both neutrino and anti-neutrino throughout almost all the energy range. Therefore, the common shift of $a_{0}$ for neutrino and anti-neutrino can mimic the effect of $a_{1}$, that is, the correlation does exist. In $5,000 \mathrm{~km} \lesssim L \lesssim 7,500 \mathrm{~km}, \frac{\lambda_{+}-\lambda_{-}}{4 E} L$ for anti-neutrino is not around either 0 or $\frac{\pi}{2}$. However, since there will not be a significant number of anti-neutrino events, in this region, the statistics will be dominated by neutrino events and hence the correlation still exists. In the region beyond $7,500 \mathrm{~km}$, the high-energy neutrino no longer follows the condition, and so the correlation will cease to hold. We conclude that there is a strong correlation between $a_{0}$ and $a_{1}$ in the case where the baseline length is less than $7,500 \mathrm{~km}$.

We would like to note one more thing. According to eqs.(5) and (6), the same relation also holds for $\nu_{e} \rightarrow \nu_{\tau}$ and $\nu_{e} \rightarrow \nu_{e}$. So, even if we utilize these channels, the correlation will not be broken.

\section{NUMERICAL CALCULATIONS}

To illustrate how the uncertainty of $a_{1}$ impairs sensitivity to the CP violation, we present the numerical results for it, including the matter profile effect and its uncertainty. In an effort to clarify the difference from former research, we show simultaneously the result without consideration of the matter profile.

First, we explain briefly the procedure for drawing the sensitivity plot: We define the test statistics,

$$
\chi^{2} \equiv \min _{\substack{\text { osc. } \\ \delta=\{0, \pi\}}}\left[\sum_{i}^{\text {bin }} \frac{\left|\bar{N}_{i}^{\text {th }} \times N_{i}^{\text {ex }}-N_{i}^{\text {th }} \times \bar{N}_{i}^{\text {ex }}\right|^{2}}{\left(\bar{N}_{i}^{\text {th }}\right)^{2} \times N_{i}^{\text {ex }}+\left(N_{i}^{\text {th }}\right)^{2} \times \bar{N}_{i}^{\text {ex }}}\right] .
$$

Here, $N^{\text {ex }}$ is the "expected number of events" for $\nu_{e} \rightarrow \nu_{\mu}$ calculated using the full-PREM matter profile with $\delta=\pi / 2$, $N^{\text {th }}$ is the "theoretical number of events", calculated with the constant matter profile or the up-to-first-mode profile with $\delta=\{0, \pi\}$, and $\bar{N}$ denotes the number of events for $\bar{\nu}_{e} \rightarrow \bar{\nu}_{\mu}$. The index $i$ stands for the energy bin. The parameters contained in $N^{\text {th }}$ and $\bar{N}^{\text {th }}$ are varied within given ambiguities. We adjust them and minimize $\chi^{2}$ to introduce the effect of the parameter correlation 2 , 19. The widths of uncertainty of the parameters concerning the atmospheric and solar neutrino experiments are expected to be narrowed by near-future experiments 20. Therefore, we assume

$$
\begin{aligned}
& \Delta(\sin \psi)=1 \%, \quad \Delta\left(\Delta m_{31}^{2}\right)=3 \%, \\
& \Delta(\sin \omega)=5 \%, \quad \Delta\left(\Delta m_{21}^{2}\right)=5 \%,
\end{aligned}
$$

and for the other parameters including the matter effect we assume some options and compare them to each other. We do not deal with systematic error. We require 
in order to claim that the hypothesis with $\delta=\{0, \pi\}$ is excluded at the $99 \%$ level of significance, where the right-hand side is the $\chi^{2}$ distribution function whose degree of freedom is the number of energy bins, not the number of parameters which fit. This is because we adopt the concept, the power of test (see appendix of Ref. [19] for more details). The reason why we use this, less familiar, concept is that we firmly believe that we must pay attention to the fact that the best-fit point suggested by an experiment is not always located on the point chosen by nature. Remind yourself how the best-fit point for the solar neutrino deficit has changed. We actually know that there were several good-fitting regions for the solar neutrino experiments which were separated from each other on the parameter plane and which were not distributed only around the best-fit point. Moreover, the best-fit point itself moved from one region to another. To discuss the feasibility of observing some quantity with an experiment, we have to consider this fact.

We also note in passing that the degree of freedom for so-called $\Delta \chi^{2}$, which is often used in an estimation of the oscillation parameters, is not the number of the parameters since, for example, two strongly correlated parameters are not independent of each other, and hence we should count them as one parameter. Indeed, as is commonly known, there are strong correlations for some parameters in the case we deal with ${ }^{\text {\% }}$. We should be more careful about what is a truly measurable quantity [21], and which quantities statistics are sensitive to [19]. For instance, if the statistics can be constructed so as to be sensitive to the CP violating effect, then the parameter is only one, Jarlskog's parameter, and the degree of freedom should be one. Strictly speaking, only when the statistics are linearly dependent on the parameters, will the degree of freedom coincide with the number of the parameters.

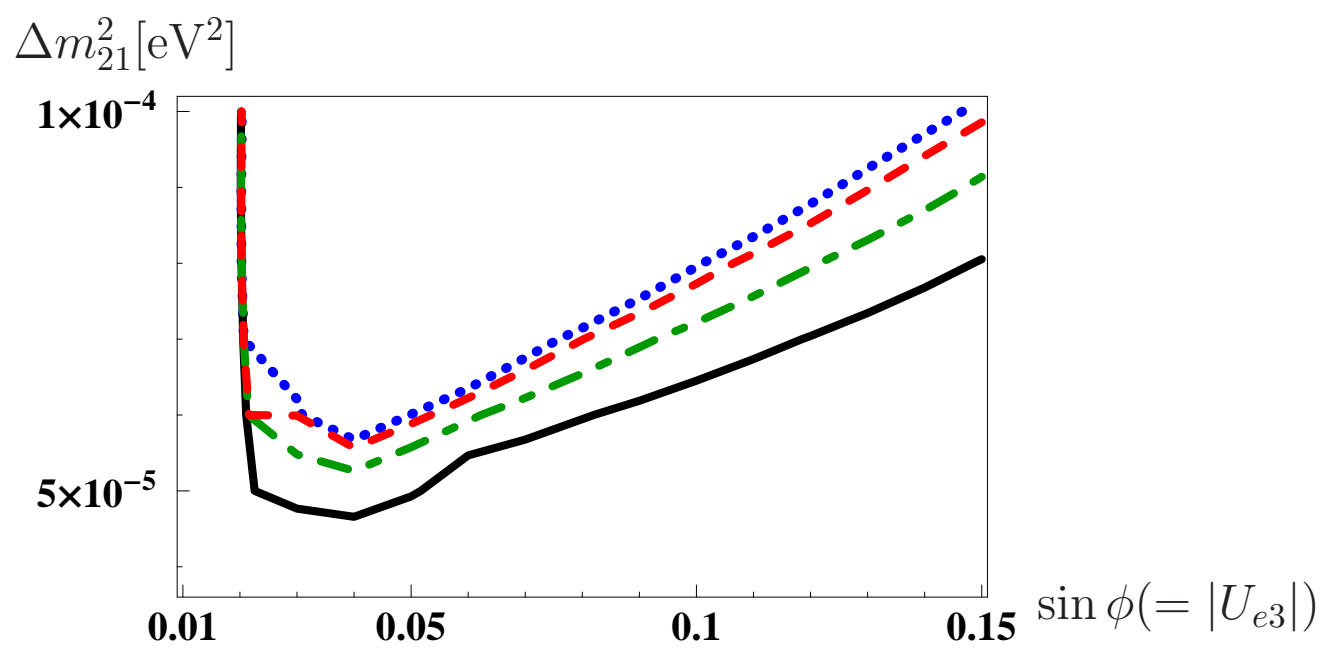

FIG. 7: Sensitivity reach for CP violation with $\delta=\pi / 2$, a baseline length of 3,000 km, and a muon energy of $30 \mathrm{GeV}$. The theoretical number of events, $N^{\text {th }}$, of the dotted curve is calculated using the up-to-first-mode profile, where the widths of parameter uncertainty are assumed to be $\Delta\left(a_{0}\right)=3 \%, \Delta\left(a_{1}\right)=200 \%$, and $\sin \phi=0 \sim 0.16$. For the other parameters, the uncertainty in eq.(14) is assumed. The solid, dash-dot and dashed curves are calculated using the constant matter profile whose uncertainties are $\Delta a_{0}=0 \%, 3 \%$ and $5 \%$, respectively, and the uncertainty for the other parameters is the same as for the dotted line.

To draw a sensitivity plot, we assume $40 \mathrm{kt}$ detector and $10^{21}$ muon decays in the neutrino factory scheme, and interpret eq.(15) to the condition for $\Delta m_{21}^{2}$ and $\sin \phi$. Figure 0 indicates the lower boundary of $\Delta m_{21}^{2}$ and $\left|U_{e 3}\right|$ $(=\sin \phi)$ to reject the hypotheses that $\delta$ is 0 and $\pi$ at a $99 \%$ level of significance, when nature adopts the value $\delta=\pi / 2$ in the case where the baseline length is $3,000 \mathrm{~km}$, muon energy is $30 \mathrm{GeV}$, the detection threshold is $5 \mathrm{GeV}$ and the width of energy bin is $2.5 \mathrm{GeV}$ (10 bin). The reference values are the same as those in Fig. 4 , except for $\Delta m_{21}^{2}$ and $\sin \phi ;$

$$
\sin \psi=1 / \sqrt{2}, \quad \Delta m_{31}^{2}=2.5 \times 10^{-3} \mathrm{eV}^{2}, \quad \sin \omega=0.5, \quad \delta=\pi / 2 .
$$

The dotted curve is calculated whilst considering the matter profile effect and its uncertainty up to the first Fourier mode in the calculation of $N^{\text {th }}$. We take $3 \%$ for $a_{0}$ and $200 \%$ for $a_{1}$ as the widths of uncertainty. The other curves are calculated assuming the constant matter profile with a different uncertainty for $a_{0}$. The solid, dash-dot, and dashed curves correspond to $\Delta\left(a_{0}\right)=0 \%, 3 \%$ and $5 \%$, respectively. In the minimization process, $\sin \phi$ in $N^{\text {th }}$ is taken as an arbitrary value between $0 \sim 0.16$, and the uncertainty of the other parameters is assumed, as in eq.(14).

The dotted curve differs from the dash-dot curve in which the same uncertainty for $a_{0}$ is assumed, but that for $a_{1}$ is not included. In contrast, it is very similar to the dashed curve whose uncertainty for $a_{0}$ is larger (5\%), but where a constant matter profile is assumed. This fact can be explained by the correlation between $a_{0}$ and $a_{1}$ eqs.(11) and (12): Uncertainty of $a_{1}$ is translated into that of $a_{0}$ through the correlation, and this extra uncertainty gives rise to an extra absorption of the signal of the CP violating effect. We know that the effect of the fake $\mathrm{CP}$ signal induced by

I Depending on the conditions, it breaks the correlation to regard the oscillation parameters as not free ones, but as restricted ones. In the numerical calculation, this effect is automatically introduced by setting the widths of the uncertainty. 
the matter effect becomes more serious as $\left|U_{e 3}\right|$ increases, so if $\left|U_{e 3}\right|$ is measured just below the current boundary, we will have to take the effect induced by the density profile of the Earth more seriously.

In the case of $L=3,000 \mathrm{~km}$, the determination of $\sin \phi$ and the reduction of its uncertainty do not contribute towards improving the sensitivity much, since the matter effect itself is large at this baseline length. Furthermore, lowering the detection threshold also hardly helps to improve the sensitivity because the number of events in the high-energy region is overwhelmingly greater than that in the low-energy region. If we can realize a lower detection threshold, an advantage can be gained with a shorter baseline length and a lower energy beam.

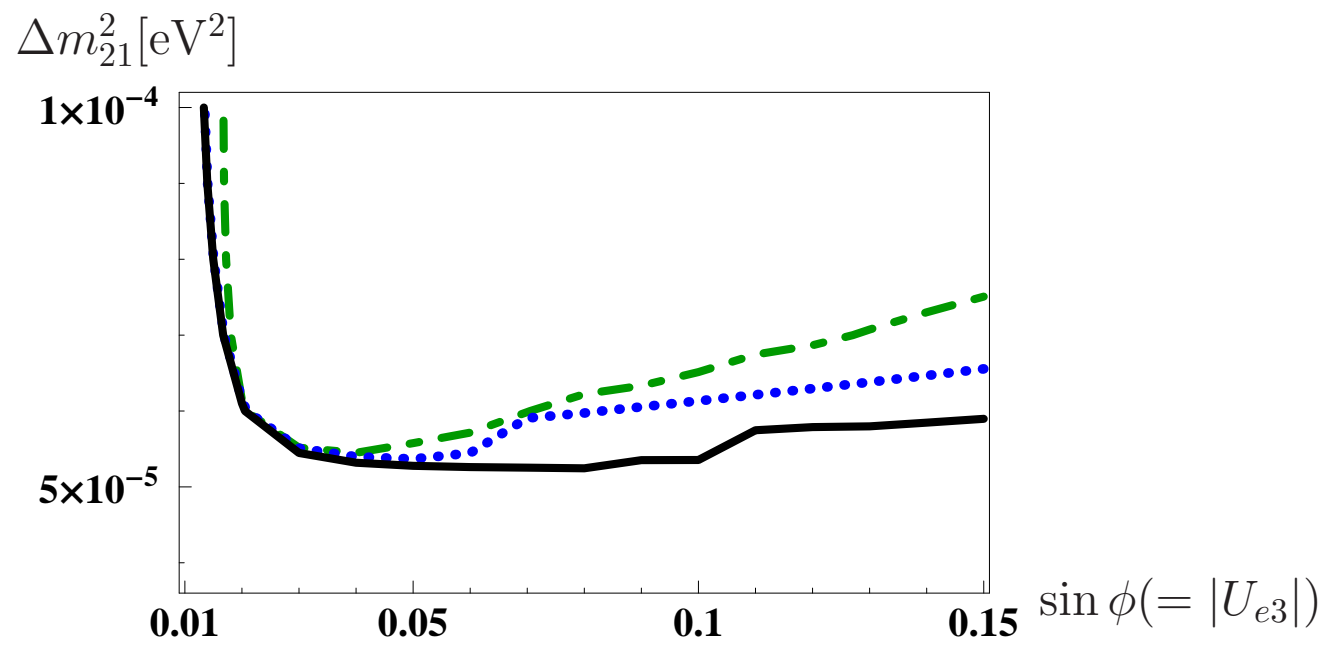

FIG. 8: Sensitivity reach for CP violation when $\delta=\pi / 2$ in the case where the baseline length is $1,000 \mathrm{~km}$, the muon energy is $11 \mathrm{GeV}$, and the detection threshold is $1 \mathrm{GeV}$. The up-to-first-mode profile is adopted for the dotted curve where its widths of uncertainty are $\Delta\left(a_{0}\right)=3 \%, \Delta\left(a_{1}\right)=200 \%, \Delta(\sin \phi)=10 \%$ and eq.(14) for the other parameters. The solid curve is calculated using the constant matter profile with $\Delta\left(a_{0}\right)=3 \%$. By comparison with Fig, 7 , it is obvious that the uncertainty of the matter effect is not serious in this situation. The dash-dot curve is calculated in a similar manner to the dotted curve, except that $\sin \phi$ varies from 0 to 0.16 . The dotted and dash-dot curves show that the uncertainty of $\sin \phi$ will play an important role in this situation if a large $\sin \phi$ is established.

Figure 8 shows the sensitivity in the case where the baseline length is $1,000 \mathrm{~km}$, the muon energy is $11 \mathrm{GeV}$ and the energy threshold is $1 \mathrm{GeV}$. In this plot, the dotted curve represents the calculation using the up-to-first-mode profile and its widths of parameter uncertainty are $\Delta\left(a_{0}\right)=3 \%, \Delta\left(a_{1}\right)=200 \%, \Delta(\sin \phi)=10 \%$, and eq. (14) for the others, the solid curve is calculated by assuming a constant matter profile with $\Delta\left(a_{0}\right)=3 \%$, and the dash-dot curve is the same as the dotted one, except that $\sin \phi$ in $N^{\text {th }}$ is assumed to be an arbitrary value between $0 \sim 0.16$.

Within the large $\left|U_{e 3}\right|$ region, the sensitivity is better than that in Fig. 0 . Furthermore, the sensitivity of this choice is more robust against the uncertainty of the matter effect than in the case of $L=3,000 \mathrm{~km}$. In contrast, although the signal of the $\mathrm{CP}$ violation is also small in the small $\left|U_{e 3}\right|$ region, the fake $\mathrm{CP}$ violation effect induced by the matter effect is suppressed more strongly than the signal of the genuine CP violation, and hence, adopting a longer baseline and higher energy option may be advantageous to the $\mathrm{CP}$ violation search.

This behavior does not come from the property of the statistics as defined by eq.(13). When the uncertainty of the constant matter parameter is assumed to be larger, the optimization, using so-called $\Delta \chi^{2}$, also suggests that a shorter baseline length and lower energy is better (see, for example O. Yasuda, in Ref[2]). The existence of this correlation tells us that even though it is said that the uncertainty of the average matter density on the baseline is well estimated, this is not the entire uncertainty of the constant matter parameter. We would like to stress that the uncertainty of the matter effect is no longer so small when we introduce the matter profile effect. Therefore, we should regard it more seriously whatever statistics we use, especially in the case where the main part of the neutrino beam path is the upper mantle and transition zone, including a large uncertainty for the density profile. We need to deal with the matter effect much more cautiously.

\section{SUMMARY AND CONCLUSION}

We pointed out that there was a very strong correlation between the constant matter parameter, $a_{0}$, and the first Fourier coefficient of the matter profile function, $a_{1}$, within a wide energy and baseline region. This fact means that the uncertainty of $a_{1}$ is translated into that of $a_{0}$, and this gives added uncertainty to $a_{0}$.

We also showed that there is a huge uncertainty in $a_{1}$. This is due to the fact that seismological Earth models include a large uncertainty for the density profile in the region, the upper mantle and the transition zone, which is $24 \sim 670 \mathrm{~km}$ in depth, and this uncertainty can cause a huge, even a few hundred percent, uncertainty for $a_{1}$. The existence of the correlation suggests that this huge uncertainty affects CP sensitivity since, due to the correlation, the uncertainty in $a_{1}$ gives added uncertainty to $a_{0}$. 
We present the sensitivity plot for the $\mathrm{CP}$ violation effect including the matter profile effect and its uncertainty. In the case where the baseline length is $3,000 \mathrm{~km}$, most of the path of the neutrino beam is occupied by the upper mantle, which includes large uncertainty. We show numerically that $200 \%$ uncertainty for $a_{1}$ can be interpreted as about $2 \%$ extra uncertainty for $a_{0}$, which should be added to the original uncertainty of $a_{0}$, and this result confirms our expectations from the correlation. This extra uncertainty makes the CP sensitivity worse, especially within the large $\left|U_{e 3}\right|$ region. A shorter baseline and lower energy option can avoid this disadvantage if the detection threshold can be lowered. On the contrary, if small $\left|U_{e 3}\right|$ is established, then a long-baseline and high-energy option may be better than a shorter baseline and lower energy, because of the statistics.

We made some comments in answer to questions about the statistics which we used. We consider the fact that the best-fit parameter suggested by the experiments is not always distributed only around the parameter chosen by nature. Therefore, to discuss the feasibility of observing the $\mathrm{CP}$ violation effect, we need to consider this fact. This led us to use the concept, the power of test.

In this study, we do not consider systematic uncertainty. Of course, in order to optimize the experimental configurations, it is necessary to take account of systematic error. However, in any case, we can conclude that we should regard the uncertainty of the Earth's matter as a more severe problem than that has so far been assumed. We need to be much more conservative when estimating the matter effect.

\section{Acknowledgments}

The authors thank the Yukawa Institute for Theoretical Physics at Kyoto University. Discussions during the YITP workshop YITP-W-02-05 on "Flavor mixing, CP violation and Origin of matter" were useful for completing this work. T. O. would like to thank W. Winter for his useful comments. The work of J. S. is supported in part by Grants-in-Aid for Scientific Research from the Ministry of Education, Science, Sports, and Culture of Japan, No. 14740168, No. 14039209 and No. 14046217. The English used in this manuscript was revised by K. Miller (Royal English Language Centre, Fukuoka, Japan).

[1] L. Wolfenstein, Phys. Rev. D17 (1978) 2369; S. P. Mikheev and A. Yu. Smirnov, Sov. J. Nucl. Phys. 42 (1985) 913 (Yad. Fiz. 42 (1985) 1441); Nuovo Cimento C9 (1986) 17; H. A. Bethe, Phys. Rev. Lett. 56 (1986) 1305; V. Barger, K. Whisnant, S. Pakvasa, and R. J. N. Phillips, Phys. Rev. D22 (1980) 2718.

[2] For example, NuFact'02 conference, http://www.hep.ph.ic.ac.uk/NuFact02/ J. Burguet-Castell, M. B. Gavela, J. J. GomezCadenas, P. Hernandez, and O. Mena, hep-ph/0207080; P. Zucchelli, Phys. Lett. B532 (2002) 166 hep-ex/0107006; A. Donini, D. Meloni, and P. Migliozzi, hep-ph/0206034; P. Huber, M. Lindner, and W. Winter, hep-ph/0204352; O. Yasuda, hep-ph/0209127, hep-ph/0203273, For a reference list, see T. Ota, J. Sato and N. Yamashita, Phys. Rev. D65 093015 hep-ph/0112329.

[3] P. I. Krastev and A. Yu. Smirnov, Phys. Lett. B226 (1989) 341.

[4] P. I. Krastev and S. T. Petcov, Phys. Lett. B205 (1988) 84; S. T. Petcov, Phys. Lett. B 434 (1998) 321; M. V. Chizhov and S. T. Petcov, Phys. Rev. D63 (2001) 073003 hep-ph/9903424; Phys. Rev. Lett. 83 (1999) 1096 hep-ph/9903399; J. Bernabeu, S. Palomares-Ruiz, A. Perez, and S. T. Petcov, Phys. Lett. B531 (2002) 90 [hep-ph/011007].

[5] E. K. Akhmedov, Nucl. Phys. B538 (1999) 25 hep-ph/9805272; Pramana 54 (2000) 47 hep-ph/9907435; Phys. Atom. Nucl. 64 (2001) 787 (Yad. Fiz. 64 (2001) 851) hep-ph/0008134; E. K. Akhmedov, A. Dighe, P. Lipari, A.Y. Smirnov, Nucl. Phys. B542 (1999) 3 hep-ph/9808270.

[6] M. Freund and T. Ohlsson, Mod. Phys. Lett. A15 (2000) 867 hep-ph/9909501; T. Ohlsson and H. Snellman, J. Math. Phys. 41 (2000) 2768, Erratum ibid. 42 (2001) 2345 hep-ph/9910546;; Phys. Lett. B474 (2000) 153 hep-ph/9912295; T. Ohlsson, Phys. Lett. B522 (2001) 280 hep-ph/0109003.

[7] I. Mocioiu and R. Shrock, Phys. Rev. D62 (2000) 053017 hep-ph/0002149.

[8] G. L. Fogli, G. Lettera, and E. Lisi, hep-ph/0112241.

[9] E. K. Akhmedov, P. Huber, M. Lindner, and T. Ohlsson, Nucl. Phys. B608(2001) 394 hep-ph/0105029

[10] T. Miura, E. Takasugi, Y. Kuno, and M. Yoshimura, Phys. Rev. D64 (2001) 013002 hep-ph/010211]; T. Miura, T. Shindou, E. Takasugi, and M. Yoshimura, Phys. Rev. D64 (2001) 073017 hep-ph/0106086.

[11] L. Shan, B. Young, and X. Zhang, hep-ph/0110414.

[12] B. Jacobsson, T. Ohlsson, H. Snellman, and W. Winter, hep-ph/0112138.

[13] T. Ohlsson and W. Winter, Phys. Lett. B512 (2001) 357 [hep-ph/0105293]; M. Lindner, T. Ohlsson, R. Tomas, and W. Winter, hep-ph/0207238.

[14] M. Koike and J. Sato, Mod. Phys. Lett. A14 (1999) 1297 hep-ph/9803212.

[15] T. Ota and J. Sato, Phys. Rev. D63 (2001) 093004 hep-ph/0011234.

[16] A. M. Dziewonski and D. L. Anderson, Phys. Earth Planet. Inter. 25 (1981) 297.

[17] B. L. N. Kennett, E. R. Engdahl, and R. Buland, Geophys. J. Int., 122 (1995) 108. http://wwwrses.anu.edu.au/seismology/ak135/intro.html

[18] R. Geller and T. Hara, hep-ph/0111342.

[19] M. Koike, T. Ota, and J.Sato, Phys. Rev. D65 053015 hep-ph0011387v3].

[20] JHF-SK Collaboration. hep-ex/0106019. For KamLand experiment, see P. Aliani, V. Antonelli, M. Picariello, and E. Torrente-Lujan, hep-ph/0207348; M. C. Gonzalez-Garcia and C. Pena-Garay, Phys. Lett. B527 (2002) 199 hep- 
ph/0111432]; H. Murayama, Phys. Rev. D65 (2002) 013012 hep-ph/0012075; V. D. Barger, D. Marfatia, and B. P. Wood, Phys. Lett. B498 (2001) 53 hep-ph/0011251.

[21] J. Sato, Nucl. Instrum. Meth. A472 (2001) 434 hep-ph/0008056. 